\title{
Mitochondrial Aging: Focus on Mitochondrial DNA Damage in Atherosclerosis - A Mini-Review
}

\author{
Igor A. Sobenin ${ }^{a, b}$ Andrey V. Zhelankin ${ }^{a, b}$ Vasily V. Sinyov ${ }^{a}$ \\ Yuri V. Bobryshev ${ }^{b, d, e}$ Alexander N. Orekhov ${ }^{b, c}$ \\ a Laboratory of Medical Genetics, Institute of Clinical Cardiology, Russian Cardiology Research and Production

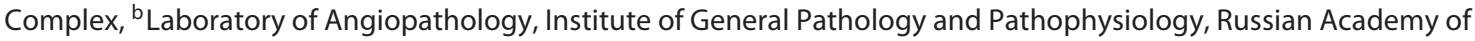 \\ Medical Sciences, and 'Institute for Atherosclerosis Research, Skolkovo Innovation Center, Moscow, Russia; ${ }^{\mathrm{d} F a c u l t y}$ \\ of Medicine, School of Medical Sciences, University of New South Wales, Sydney, N.S.W., and 'School of Medicine, \\ University of Western Sydney, Campbelltown, N.S.W., Australia
}

\author{
Key Words \\ Atherosclerosis · Aging · Mitochondria $\cdot$ Mitochondrial \\ DNA $\cdot$ Cell senescence $\cdot$ Mutations
}

portant role in the development of atherosclerosis and which are, at the same time, also markers of mitochondrial aging and cell senescence. (c) 2014 S. Karger AG, Basel

\begin{abstract}
Atherosclerosis is a complex disease which can be described as an excessive fibrofatty, proliferative, inflammatory response to damage to the artery wall involving several cell types such as smooth muscle cells, monocyte-derived macrophages, lymphocytes, dendritic cells and platelets. On the other hand, atherosclerosis is a typical age-related degenerative pathology, which is characterized by signs of cell senescence in the arterial wall including reduced cell proliferation, irreversible growth arrest and apoptosis, increased DNA damage, the presence of epigenetic modifications, shortening of telomere length and mitochondrial dysfunction. The most prominent characteristics of mitochondrial aging are their structural alterations and mitochondrial DNA damage. The mechanisms of mitochondrial genome damage in the development of chronic age-related diseases such as atherosclerosis are not yet well understood. This review focuses on the latest findings from studies of those mutations of the mitochondrial genome which may play an im-
\end{abstract}

Atherosclerosis, by far the greatest killer in modern society, is a complex disease which can be described as an excessive fibrofatty, proliferative, inflammatory response to damage to the artery wall involving several cell types such as smooth muscle cells, monocyte-derived macrophages, lymphocytes, dendritic cells and platelets $[1,2]$. Clinical manifestations of atherosclerosis, i.e. mainly coronary artery disease and stroke, are the leading causes of death in all economically developed countries, accounting for up to $65 \%$ of total mortality [3]. Many factors appear to contribute to the development of atherosclerosis, including alterations in plasma lipid and lipoprotein levels, platelet function, clotting factors, arterial smooth muscle cell metabolism and blood pressure regulation. However, the precise mechanisms of atherogenesis are still unclear, even if it is well known that the deposition of intracellular lipids, mainly free and esterified cholesterol, as well as subsequent foam cell formation are the most

\section{KARGER 125\%}

(c) 2014 S. Karger AG, Basel

0304-324X/14/0614-0343\$39.50/0

E-Mail karger@karger.com

www.karger.com/ger
Yuri V. Bobryshev

Faculty of Medicine, School of Medical Sciences

University of New South Wales

Sydney, NSW 2052 (Australia)

E-Maily.bobryshev@unsw.edu.au 
typical features of early atherosclerotic lesion development [4]. Modified low-density lipoprotein (LDL) is generally thought to be the source of accumulating lipids [5]. Intracellular lipid deposition may act as a trigger mechanism for the development of advanced atherosclerotic lesions, implying an excessive production of connective tissue matrix components as well as, possibly, cellular proliferation and inflammatory reactions $[4,6-8]$. The subsequent formation of advanced atherosclerotic lesions finally results in widespread clinical manifestations of atherosclerosis.

Atherosclerosis may also be regarded as a typical agerelated degenerative pathology, which is characterized by signs of cell senescence in the arterial wall, including reduced cell proliferation, irreversible growth arrest and apoptosis, increased DNA damage, the presence of epigenetic modifications, shortening of telomere length and mitochondrial dysfunction [9]. In elderly subjects, characteristic changes occur in several cell types in the arterial intima, including smooth muscle $[10,11]$, inflammatory [12] and endothelial cells [13]. The signs of cellular aging in atherosclerotic plaques appear as changes of proliferative potential in cells expressing markers of cellular aging, increasing susceptibility to apoptosis, an increased degree of mitochondrial DNA (mtDNA) damage as well as extensive telomere shortening and mitochondrial dysfunction.

Changes in mitochondria are well known to occur during aging [14-16]. The most prominent signs of mitochondrial aging are the structural alterations to this organelle as well as mtDNA damage [17-19]. mtDNA has been extensively studied since its discovery in 1963, and particularly intensive studies were conducted when an association of mtDNA mutations with the diseases of oxidative phosphorylation was revealed. As a rule, each cell contains tens to hundreds of mitochondria, and each mitochondrion contains its own copy of the genome, several copies in fused mitochondria. In the absence of mtDNA mutations, all mitochondria in the cell, tissue or whole organism should have the same variant of mtDNA, the situation being described as 'homoplasmy'. Somatic mutations of mtDNA frequently arise during the life of an individual, and, at the same time, many mutations of the mitochondrial genome can be inherited through the maternal lineage and then multiply by dividing organelles containing mutant DNA. The inheritance of mtDNA mutations is rather random, due to the asymmetric manner of the distribution of mtDNA copies containing the mutant allele between divided cells. In this case, the phenomenon of a parallel existence of mutant and wild-type
mtDNA within one cell or tissue is observed, as described by the term 'heteroplasmy'. Thus, heteroplasmy is defined as the presence of a mixture of more than one type of genome in organelles. The penetrance and expression of mtDNA mutations vary widely and depend on many factors but mainly on the genotype and the level of heteroplasmy. Such specific characteristics of mtDNA allow the accumulation of mutations, thus forming a carrier phenotype. The level of heteroplasmy in cells of the same organism can vary considerably. Mutations occurring in the mitochondrial genome may be a probable cause of the development of various noninfectious chronic human diseases including atherosclerosis and its clinical manifestations, hypertension, type 2 diabetes mellitus and obesity [20-26].

The matter of the hereditary or acquired nature of mtDNA mutations is still debated. The mitochondrial genome is characterized by evident instability, with the estimated rate of mutations at least 5- to 15 -fold higher than that in the nuclear genome. The high mutation rate determines the high level of variability of $\mathrm{mtDNA}$ as well as the emergence of somatic mtDNA mutations during aging [27]. The proportion of mutant mtDNA copies bearing both inherited and somatic mutations may change with aging due to an unequal distribution of mitochondrial genotypes between cells during cytokinesis (vegetative segregation) or in nondividing cells during $\mathrm{mtDNA}$ replication. The resulting increase in the degree of heteroplasmy is considered to be the result of the clonal expansion of low-level inherited variants, which occurs due to the preferential replication of mtDNA carrying certain types of mutations [28].

However, the mechanisms of mitochondrial genome damage in the development of chronic age-related diseases such as atherosclerosis are not well understood. There is very little data yet showing a causal relationship between mtDNA damage and atherosclerosis. In general, the role of genetic factors in the development and progression of atherosclerotic disease is well documented; nevertheless, numerous polymorphisms of the nuclear genome considered as genetic risk factors for atherosclerosis can help explaining only a few percentages of the variability of clinical manifestations of atherosclerosis, such as coronary heart disease (CHD) [29]. In contrast, mutations of the mitochondrial genome may play a pathogenic role in the formation of atherosclerotic lesions in arteries, causing various defects in the protein chains of some energy-generating enzymes and transfer RNA (tRNA) synthesized directly in the mitochondria [30]. This may lead to a decrease in the concentration of 
these enzymes and tRNAs, or to total mitochondrial dysfunction, which contributes to the development of oxidative stress, deterioration in ATP production and accelerated development of atherosclerosis. The electron transport chain constantly produces superoxide radical anions, which, in the case of mitochondrial dysfunction, cause the escape of electrons that readily form hydroxyl radicals and hydrogen peroxide from superoxide. These extremely reactive oxygen species (ROS) are risk factors for atherosclerosis associated with lipid and protein oxidation in the vascular wall. ROS formation may trigger a cascade of events such as modification of LDL, inflammation, cellular apoptosis and endothelial injury. In atherosclerotic lesions, advanced ROS production positively correlates with multiple mitochondrial abnormalities including impaired mitochondrial dynamics, mitochondrial dysfunction, altered oxidative phosphorylation capacity, the induction of mitochondrion-dependent apoptosis and other pathologic changes [31]. The close proximity of mtDNA to the harmful ROS makes it vulnerable to oxidative attack. Increased oxidative stress induced by ROS results in direct damage to the mtDNA [32] as well as in a reduction in copy numbers of $\mathrm{mtDNA}$ in peripheral blood mononuclear cells [33]; a lower copy number of mtDNA correlates with the severity of independent risk factors of atherosclerosis such as hyperlipidemia [34]. In macrophages, a reduced ability of mitochondria to utilize oxidized lipids can accelerate the transformation of macrophages to foam cells and therefore participate in atherogenesis.

Nowadays, it is appreciated that age-related changes in mitochondria are associated with a decline in mitochondrial function. With advanced age, mitochondria are characterized by impaired function such as lowered oxidative capacity, reduced oxidative phosphorylation, decreased ATP production, a significant increase in ROS generation and a diminished antioxidant defense; mitochondrial biogenesis declines with age due to alterations in mitochondrial dynamics and the inhibition of mitophagy, an autophagy process that removes dysfunctional mitochondria [35]. However, the recent findings by Kennedy et al. [36] demonstrate that an age-related increase in somatic mitochondrial mutations is inconsistent with oxidative damage.

Since age is one of the most important determinants of cardiovascular health, a possible explanation of the vascular senescence process is mitochondrial damage and dysfunction. Rizza et al. [37] have hypothesized that metabolomic profiling would identify biomarkers predicting major cardiovascular events in elderly people, and they

Mitochondrial Aging in Atherosclerosis have demonstrated that age-related mitochondrial dysfunction as evaluated by metabolomic profiling is associated with major cardiovascular events independently of standard predictors.

It is known that DNA damage is present in both genomic DNA and mtDNA in atherosclerosis. However, whether DNA damage itself promotes atherosclerosis, or is simply a by-product of the risk factors that promote atherosclerosis, is unknown. Mercer et al. [38] have demonstrated that DNA damage links mitochondrial dysfunction to atherosclerosis and the metabolic syndrome. They have studied apolipoprotein E (ApoE) knockout mice that were also haploinsufficient for the protein kinase ataxia telangiectasia mutated (ATM), which coordinates DNA repair, and have shown that $\operatorname{ATM}(+/-) /$ ApoE(-/-) mice developed accelerated atherosclerosis and multiple features of the metabolic syndrome including hypertension, hypercholesterolemia, obesity, steatohepatitis and glucose intolerance. Metabolomic screening of $\operatorname{ATM}(+/-) / \operatorname{ApoE}(-/-)$ mouse tissues identified metabolic changes compatible with mitochondrial defects, with increased $\beta$-hydroxybutyrate but reduced lactate, reduced glucose and alterations in multiple lipid species. Besides, ATM(+/-)/ApoE(-/-) mice had an increased frequency of a mouse mitochondrial 'common' deletion equivalent and reduced mitochondrial oxidative phosphorylation [38].

Oxidative stress associated with cardiovascular disease risk factors contributes to disease development. However, less is known about whether specific subcellular components play a role in disease susceptibility. Harrison et al. [39] have tried to determine whether altered mitochondrial oxidant production directly influences atherogenic susceptibility and response in primary cells to atherogenic factors such as tumor necrosis factor- $\alpha$; they assessed atherosclerotic lesion formation, oxidant stress and mtDNA damage in male ApoE knockout mice with normal and decreased levels of mitochondrial superoxide dismutase 2 exposed to environmental tobacco smoke or filtered air and have demonstrated that endogenous mitochondrial oxidative stress is an important cardiovascular risk factor that can modulate atherogenesis and cytokine-induced endothelial cell oxidant generation [39].

The studies performed by Ding et al. [40] have shown that oxidized LDL induced autophagy and damaged mtDNA, leading to Toll-like receptor 9 (TLR9) expression. Lectin-like oxidized LDL scavenger receptor 1 (LOX-1) antibody or the ROS inhibitor apocynin attenuated autophagy mediated by oxidized LDL, mtDNA damage and TLR9 expression, suggesting that these events are 
LOX-1- and ROS-dependent phenomena. Experiments using small interfering (si)RNA to DNase II indicated that DNase II digests mtDNA to protect the tissue from inflammation. Ding et al. [40] also found intense autophagy, TLR9 expression and inflammatory signals in the aortas of LDL receptor knockout mice fed high cholesterol diets; a deletion of LOX-1 attenuated autophagy, TLR9 expression as well as CD45 and CD68. The researchers have stated that mtDNA damaged through oxidative stress and escaping autophagy induces a potent inflammatory response in atherosclerosis [40].

Mitochondrial oxidative stress has been shown to correlate with the progression of human atherosclerosis. Wang et al. [41] have demonstrated that macrophage mitochondrial oxidative stress promotes atherosclerosis and nuclear factor- $\mathrm{kB}$-mediated inflammation in macrophages. They have validated Western diet-fed LDL receptor knockout mice as a model of a human mitochondrial oxidative stress-to-atherosclerosis relation by showing that oxidative mtDNA damage, a marker of oxidative stress in lesional macrophages, correlates with aortic root lesion development. When oxidative stress was successfully suppressed in these mice, a significant reduction in aortic root lesional area was observed [41].

However, it has been demonstrated that mtDNA damage can promote atherosclerosis independently of ROS through effects on smooth muscle cells and monocytes, this effect being correlated with higher-risk plaques in humans [42]. Thus, mtDNA damage occurred early in the vessel wall in ApoE knockout mice before significant atherosclerosis developed. The defects in mtDNA were also identified in circulating monocytes and the liver and were associated with mitochondrial dysfunction. To determine whether mtDNA damage directly promotes atherosclerosis, Yu et al. [42] have studied ApoE knockout mice deficient for mitochondrial polymerase- $\gamma$ proofreading activity. These mice showed extensive mtDNA damage and defects in oxidative phosphorylation, but no increase in ROS, as well as increased atherosclerosis associated with impaired proliferation and apoptosis of vascular smooth muscle cells and hyperlipidemia. To examine mtDNA damage in human atherosclerosis, Yu et al. [42] assessed mtDNA adducts in plaques and in leukocytes from patients who had undergone a virtual histology intravascular ultrasound characterization of coronary plaques, and they demonstrated that human atherosclerotic plaques showed increased mtDNA damage compared with normal vessels; in contrast, leukocyte mtDNA damage was associated with higher-risk plaques but not plaque burden.
Since LOX-1 and mtDNA damage play a key role in a variety of cardiovascular diseases including atherosclerosis, hypertension and inflammation, Ding et al. [43] have suggested that damaged mtDNA could trigger autophagy and NLR family, pyrin domain-containing 3 (NLRP3) inflammasome activation. In order to examine this hypothesis, cultured human THP1 macrophages exposed to lipopolysaccharide were applied to study the link between LOX-1, mtDNA damage, autophagy and NLRP3 inflammasome expression. It has been demonstrated that lipopolysaccharide markedly induced LOX-1 expression, ROS generation, autophagy, mtDNA damage and NLRP3 inflammasome expression. LOX-1 inhibition with a binding antibody or siRNA inhibited ROS generation, autophagy and mtDNA damage and decreased NLRP3 inflammasome expression. Both ROS inhibitors and an autophagy inducer decreased the expression of NLRP3. On the other hand, an autophagy inhibitor enhanced NLRP3 inflammasome expression. Knockdown of DNase II inhibited autophagy and NLRP3 inflammasome expression. The relationship between LOX-1, ROS, mtDNA damage, autophagy and NLRP3 inflammasome activation in primary macrophages was confirmed [43].

There is a substantial experimental and publication bias towards data derived from studies of animal models, especially ApoE knockout mice. The evidence from human studies is incomparable to that from animal studies, both in methodology and endpoints. Therefore, it is not possible to make direct comparisons. Indeed, the bulk of studies has been performed on animal models of atherosclerosis, which are still far from reflecting human atherosclerosis, and it is impossible to perform direct interpolations to human pathology. Wild-type mice do not develop atherosclerosis, for several reasons: in particular, they have very low levels of LDL, their lipoprotein metabolism and turnover is quite different from that of humans, and there is no intimal layer in the arteries, which is where atherosclerosis should develop. The development of atherosclerosis-like (more correctly, xanthomatosis-like) changes is possible in ApoE knockout or LDL receptor knockout mice fed high-cholesterol diets, a genetic and behavioral situation hardly possible in humans. On the other hand, there exists a consensus that mtDNA damage accumulation occurs in humans with aging, and mtDNA damage has been reported to be associated with various chronic diseases in human studies. It is notable that the vast majority of the studies in humans used DNA isolated from circulating blood cells, which is easily available biological material. This may invoke a methodological bias due to the existence of between-tissue mitochondrial genome variation [44]. 
Special attention should be devoted to the parallelism between mitochondrial dysfunction, mtDNA damage and telomere shortening, which are generally attributed to cellular senescence [9]. One should consider the crucial differences in the pathophysiological essence of these markers of cellular aging. While telomere shortening reflects the lower ability of senescent tissues to regenerate and telomeres exist in nuclear DNA but not mtDNA, damaged mtDNA appears in tissues due to the deleterious action of different agents like ROS or because of the selective migration and accumulation of cells bearing the mutant mitochondrial genome. Mitochondrial dysfunction may be only partially explained by mtDNA damage. In studies on humans, it has been demonstrated that the relative telomere length is shortened in atherosclerotic patients compared with age- and sex-matched, apparently healthy subjects $[45,46]$. However, there is still no evidence on the casual relationship between telomere length and atherosclerosis, and this marker of cellular senescence may be a bystander that changes in parallel with atherosclerosis progression. The studies on humans and on mice have highlighted the importance of short telomeres and impaired mitochondrial function in driving age-related functional decline in the arteries. The recent studies on telomerase-deficient mice have provided the evidence for the link between these two processes. Telomere dysfunction induces a profound p53-dependent repression of the master regulators of mitochondrial biogenesis and function, i.e. peroxisome proliferator-activated receptor- $\gamma$ coactivators, which leads to bioenergetic compromise due to impaired oxidative phosphorylation and ATP generation. This metabolic axis integrates many factors linked to aging, including increased DNA damage and p53 activation as well as mitochondrial and metabolic dysfunction, and provides a molecular basis for how dysfunctional telomeres can precipitate arterial aging [47].

Several studies demonstrated the monoclonality of the amplification of vascular smooth muscle cells in atherosclerotic lesions $[48,49]$. Thus, the monoclonal origin of atherosclerotic plaques may represent a likely mechanism of expansion of cells containing mtDNA with a high content of mutated $\mathrm{mtDNA}$ within an atherosclerotic plaque. To date, only few studies have evaluated whether heteroplasmic mutations of mtDNA are associated with atherosclerosis. In two studies, the mtDNA mutation del4977 (a deletion of a nearly $5-\mathrm{kb} \mathrm{mtDNA}$ region containing 5 genes for tRNA and 7 genes for respiratory chain enzymes) was assessed, but no significant difference in heteroplasmy levels was found between healthy subjects and patients with coronary atherosclerosis $[22,50]$. On the other hand, the frequency of this mutation was 5-fold higher in atherosclerotic patients than in apparently healthy individuals [22]. Mutation T16189C, occurring in the hypervariable D-loop of the control region of $\mathrm{mtDNA}$, has attracted research interest because of its suspected association with various multifactorial diseases. In a study performed on 482 patients with coronary artery disease, 505 type 2 -diabetic patients and 1,481 apparently healthy individuals, it has been demonstrated that the prevalence of T16189C mutations was significantly higher in patients with coronary artery disease as well as in diabetics [51].

Recently, the results of a comprehensive analysis of 40 mtDNA mutations in atherosclerotic tissues have been reported. Ten mutations were characterized by the levels of heteroplasmy significantly differing in atherosclerotic lesions from those in normal aortas [52, 53]. Mutations of $\mathrm{mtDNA}$ associated with lipofibrous plaques have been found in mitochondrial genes encoding rRNA12S, tRNA $^{\text {Leu }}$ (UUR recognition codon), tRNA ${ }^{\text {Leu }}$ (CUN recognition codon), subunits of 1, 2, 5 and $6 \mathrm{NADH}$-dehydrogenase and cytochrome B. From 29 up to $86 \%$ of the aortic samples showed a significant difference between atherosclerotic plaques and unaffected tissue with respect to the level of heteroplasmy for each mutation [53]. For the mtDNA mutations A1555G, C3256T, T3336C, G13513A and G15059A, the mean level of heteroplasmy in atherosclerotic intimal homogenates was different from that in the unaffected tissue; the integral mutation burden of mtDNA in intimal tissue independently explained not less than $14 \%$ of the variability of atherosclerosis [54]. mtDNA mutations have been suggested to interrelate with the structural alterations to mitochondria in atherosclerosis (online suppl. fig. 1; for all online suppl. material, see www.karger.com/doi/10.1159/000368923) $[30,54]$.

In a cross-sectional clinical study performed on 191 subjects, $24 \%$ of whom had CHD, the association between the level of heteroplasmy for the mutation C3256T, quantitatively measured by pyrosequencing in human white blood cells, and the extent of carotid atherosclerosis, estimated by high-resolution B-mode ultrasonography of carotids followed by measurement of carotid intima-media thickness (cIMT), was studied [55]. A highly significant relationship between C3256T heteroplasmy level and predisposition to atherosclerosis (as estimated by cIMT measurement) was revealed. In individuals with a low predisposition to atherosclerosis, the mean level of C3256T heteroplasmy was $16.8 \%$ as compared to $23.8 \%$ 
in moderately predisposed subjects and to 25.2 and $28.3 \%$ in significantly and highly predisposed subjects, respectively [55].

The association of mitochondrial genetic variation with the severity of carotid atherosclerosis, as assessed by cIMT and the presence of CHD, was further analyzed [56]. Significant correlations were found between cIMT and the levels of heteroplasmy for the C3256T, T3336C, G12315A, G13513A and G15059A mutations of mtDNA. Additionally, the levels of heteroplasmy for the mutations C3256T, T3336C, G12315A, G13513A, G14459A, G14846A and G15059A correlated significantly with the size of atherosclerotic plaques visualized in any segment of carotid arteries. This in-depth statistical analysis has supported the assumption of a significant association of atherosclerosis and CHD with the heteroplasmy level of mtDNA mutations. The model which included only conventional risk factors such as age, gender, diabetes, hypertension and triglycerides as well as LDL and HDL cholesterol explained $24.5 \%$ of the cIMT variability, whereas the model which also included the levels of heteroplasmy for mtDNA mutations reached a significantly better explanatory level of $33.5 \%$. The inclusion of mutations also added a significant contribution to the increase in explanatory power for the diagnosis of the presence of atherosclerotic plaques in any visualized segment of carotids [56]. However, it should be noted that determining the true muta- tion load of mtDNA is an extremely difficult task, and the mitochondrial disease field dealing with patients with high levels of heteroplasmy still encounters large variations if different laboratories measure heteroplasmy levels in the same samples. Therefore, till now, a true causal relationship has not been proven between mtDNA mutations and atherosclerosis.

Taken together, the above-described findings indicate that mutations of the mitochondrial genome play a substantial role in the development of atherosclerosis, being also the markers of mitochondrial aging and cell senescence. However, the impact of certain mtDNA mutations on atherogenesis is widely unknown. Obviously, more studies are required which focus on the assessment of new heteroplasmic mtDNA mutations affecting mitochondrial function in relation to atherosclerosis.

\section{Acknowledgment}

The authors wish to acknowledge the support from the Russian Scientific Foundation (grant No. 14-14-01038).

\section{Disclosure Statement}

The authors report no conflict of interest.

\section{References}

1 Schwartz CJ, Valente AJ, Sprague EA: A modern view of atherogenesis. Am J Cardiol 1993; 71:9B-14B.

2 Libby P, Ridker PM, Hansson GK: Progress and challenges in translating the biology of atherosclerosis. Nature 2011;473:317-325.

$\checkmark 3$ Wood D, De Backer G, Faergeman O, Graham I, Mancia G, Pyörälä K: Prevention of coronary heart disease in clinical practice: recommendations of the Second Joint Task Force of European and other Societies on Coronary Prevention. Atherosclerosis 1998;140:199-270.

4 Orekhov AN, Tertov VV, Novikov ID, Krushinsky AV, Andreeva ER, Lankin VZ, Smirnov $\mathrm{VN}$ : Lipids in cells of atherosclerotic and uninvolved human aorta. 1. Lipid composition of aortic tissue and enzyme-isolated and cultured cells. Exp Mol Pathol 1985;42:117-137.

$>5$ Miller YI, Choi SH, Fang L, Tsimikas S: Lipoprotein modification and macrophage uptake: role of pathologic cholesterol transport in atherogenesis. Subcell Biochem 2010;51: 229-251.

-6 Tertov VV, Orekhov AN, Ryong LH, Smirnov $\mathrm{VN}$ : Intracellular cholesterol accumulation is accompanied by enhanced proliferative activity of human aortic intimal cells. Tissue Cell 1988;20:849-854.

7 Orekhov AN, Tertov VV, Kudryashov SA, Smirnov VN: Triggerlike stimulation of cholesterol accumulation and DNA and extracellular matrix synthesis induced by atherogenic serum or low density lipoprotein in cultured cells. Circ Res 1990;66:311-320.

-8 Bobryshev YV, Andreeva ER, Mikhailova IA, Andrianova IV, Moisenovich MM, Khapchaev S, Agapov II, Sobenin IA, Lusta KA, Orekhov AN: Correlation between lipid deposition, immune-inflammatory cell content and MHC class II expression in diffuse intimal thickening of the human aorta. Atherosclerosis 2011;219:171-183.

$>9$ Wang C, Bennett M: Aging and atherosclerosis: mechanisms, functional consequences, and potential therapeutics for cellular senescence. Circ Res 2012;111:245-259.

10 Stemerman M, Weinstein R, Rowe J, Maciag T, Fuhro R, Gardner R: Vascular smooth muscle cell growth kinetics in vivo in aged rats. Proc Natl Acad Sci USA 1982;79:3863-3866.
11 Hariri RJ, Hajjar DP, Coletti D, Alonso DR, Weksler ME, Rabellino E: Aging and arteriosclerosis: cell cycle kinetics of young and old arterial smooth muscle cells. Am J Pathol 1988;131:132-136.

12 Chung HY, Sung B, Jung KJ, Zou Y, Yu BP: The molecular inflammatory process in aging. Antioxid Redox Signal 2006;8:572-581.

$\checkmark 13$ Gerhard M, Roddy MA, Creager SJ, Creager MA: Aging progressively impairs endothelium-dependent vasodilation in forearm resistance vessels of humans. Hypertension 1996; 27:849-853.

14 Horan MP, Cooper DN: The emergence of the mitochondrial genome as a partial regulator of nuclear function is providing new insights into the genetic mechanisms underlying agerelated complex disease. Hum Genet 2014; 133:435-458.

15 Damas J, Samuels DC, Carneiro J, Amorim A, Pereira F: Mitochondrial DNA rearrangements in health and disease - a comprehensive study. Hum Mutat 2014;35:1-14.

16 Lee HC, Wei YH: Mitochondria and aging. Adv Exp Med Biol 2012;942:311-327. 
17 Wei YH: Oxidative stress and mitochondrial DNA mutations in human aging. Proc Soc Exp Biol Med 1998;217:53-63.

- 18 Lenaz G, Baracca A, Fato R, Genova ML, Solaini G: New insights into structure and function of mitochondria and their role in aging and disease. Antioxid Redox Signal 2006;8: 417-437.

19 Yao YG, Kajigaya S, Feng X, Samsel L, McCoy JP Jr, Torelli G, Young NS: Accumulation of mtDNA variations in human single CD34+ cells from maternally related individuals: effects of aging and family genetic background. Stem Cell Res 2013;10:361-370.

20 Andreassi MG: Coronary atherosclerosis and somatic mutations: an overview of the contributive factors for oxidative DNA damage. Mutat Res 2003;543:67-86.

21 Nomiyama T, Tanaka Y, Piao L, Hattori N, Uchino H, Watada H, Kawamori R, Ohta S: Accumulation of somatic mutation in mitochondrial DNA and atherosclerosis in diabetic patients. Ann NY Acad Sci 2004;1011:193204.

22 Botto N, Berti S, Manfredi S, Al-Jabri A, Federici $\mathrm{C}$, Clerico A, Ciofini E, Biagini A, Andreassi MG: Detection of mtDNA with $4977 \mathrm{bp}$ deletion in blood cells and atherosclerotic lesions of patients with coronary artery disease. Mutat Res 2005;570:81-88.

23 Marian AJ: Mitochondrial genetics and human systemic hypertension. Circ Res 2011; 108:784-786.

24 Ding Y, Xia B, Yu J, Leng J, Huang J: Mitochondrial DNA mutations and essential hypertension. Int J Mol Med 2013;32:768-774.

25 Avital G, Buchshtav M, Zhidkov I, Tuval Feder J, Dadon S, Rubin E, Glass D, Spector TD, Mishmar D: Mitochondrial DNA heteroplasmy in diabetes and normal adults: role of acquired and inherited mutational patterns in twins. Hum Mol Genet 2012;21:4214-4224.

26 Bhopal RS, Rafnsson SB: Could mitochondrial efficiency explain the susceptibility to adiposity, metabolic syndrome, diabetes and cardiovascular diseases in South Asian populations? Int J Epidemiol 2009;38:1072-1081.

$\checkmark 27$ Wallace DC: Colloquium paper: bioenergetics, the origins of complexity, and the ascent of man. Proc Natl Acad Sci USA 2011;107 (suppl 2):8947-8953.

28 Wallace DC: Mitochondrial DNA mutations in disease and aging. Environ Mol Mutagen 2010;51:440-450.

29 John PA, Ioannidis MD: Prediction of cardiovascular disease outcomes and established cardiovascular risk factors by genome-wide association markers. Circ Cardiovasc Genet 2009;2:7-15.

- 30 Sobenin IA, Chistiakov DA, Bobryshev YV, Postnov AY, Orekhov AN: Mitochondrial mutations in atherosclerosis: new solutions in research and possible clinical applications. Curr Pharm Des 2013;19:5942-5953.

-31 Davidson SM, Duchen MR: Endothelial mitochondria: contributing to vascular function and disease. Circ Res 2007;100:1128-1141.
32 Wu LL, Chiou CC, Chang PY, Wu JT: Urinary 8-OHdG: a marker of oxidative stress to DNA and a risk factor for cancer, atherosclerosis and diabetics. Clin Chim Acta 2004;339:1-9.

33 Wang YC, Lee WC, Liao SC, Lee LC, Su YJ, Lee CT, Chen JB: Mitochondrial DNA copy number correlates with oxidative stress and predicts mortality in nondiabetic hemodialysis patients. J Nephrol 2011;24:351-358.

34 Liu CS, Kuo CL, Cheng WL, Huang CS, Lee $\mathrm{CF}$, Wei YH: Alteration of the copy number of mitochondrial DNA in leukocytes of patients with hyperlipidemia. Ann NY Acad Sci 2005;1042:70-75.

35 Chistiakov DA, Sobenin IA, Revin VV, Orekhov AN, Bobryshev YV: Mitochondrial aging and age-related dysfunction of mitochondria. Biomed Res Int 2014;2014:238463.

36 Kennedy SR, Salk JJ, Schmitt MW, Loeb LA: Ultra-sensitive sequencing reveals an agerelated increase in somatic mitochondrial mutations that are inconsistent with oxidative damage. PLoS Genet 2013;9:e1003794.

-37 Rizza S, Copetti M, Rossi C, Cianfarani MA, Zucchelli M, Luzi A, Pecchioli C, Porzio O, Di Cola G, Urbani A, Pellegrini F, Federici M: Metabolomics signature improves the prediction of cardiovascular events in elderly subjects. Atherosclerosis 2014;232:260-264.

38 Mercer JR, Cheng KK, Figg N, Gorenne I, Mahmoudi M, Griffin J, Vidal-Puig A, Logan A, Murphy MP, Bennett M: DNA damage links mitochondrial dysfunction to atherosclerosis and the metabolic syndrome. Circ Res 2010;107:1021-1031

- 39 Harrison CM, Pompilius M, Pinkerton KE, Ballinger SW: Mitochondrial oxidative stress significantly influences atherogenic risk and cytokine-induced oxidant production. Environ Health Perspect 2011;119:676-681.

40 Ding Z, Liu S, Wang X, Khaidakov M, Dai Y, Mehta JL: Oxidant stress in mitochondrial DNA damage, autophagy and inflammation in atherosclerosis. Sci Rep 2013;3:1077.

41 Wang Y, Wang GZ, Rabinovitch PS, Tabas I: Macrophage mitochondrial oxidative stress promotes atherosclerosis and nuclear factor$\mathrm{\kappa B}$-mediated inflammation in macrophages. Circ Res 2014;114:421-433.

42 Yu E, Calvert PA, Mercer JR, Harrison J, Baker L, Figg NL, Kumar S, Wang JC, Hurst LA, Obaid DR, Logan A, West NE, Clarke MC, Vidal-Puig A, Murphy MP, Bennett MR: Mitochondrial DNA damage can promote atherosclerosis independently of reactive oxygen species through effects on smooth muscle cells and monocytes and correlates with higher-risk plaques in humans. Circulation 2013; 128:702-712.

43 Ding Z, Liu S, Wang X, Dai Y, Khaidakov M, Deng X, Fan Y, Xiang D, Mehta JL: LOX-1, mtDNA damage, and NLRP3 inflammasome activation in macrophages: implications in atherogenesis. Cardiovasc Res 2014;103:619628.
44 Lee HY, Chung U, Park MJ, Yoo JE, Han GR, Shin KJ: Differential distribution of human mitochondrial DNA in somatic tissues and hairs. Ann Hum Genet 2006;70(pt 1):59-65.

-45 Kroenke CH, Pletcher MJ, Lin J, Blackburn E, Adler N, Matthews K, Epel E: Telomerase, telomere length, and coronary artery calcium in black and white men in the CARDIA study. Atherosclerosis 2012;220:506-512.

46 Raschenberger J, Kollerits B, HammererLercher A, Rantner B, Stadler M, Haun M, Klein-Weigel P, Fraedrich G, Kronenberg F: The association of relative telomere length with symptomatic peripheral arterial disease: results from the CAVASIC study. Atherosclerosis 2013;229:469-474.

47 Moslehi J, DePinho RA, Sahin E: Telomeres and mitochondria in the aging heart. Circ Res 2012;110:1226-1237.

48 Chung IM, Schwartz SM, Murry CE: Clonal architecture of normal and atherosclerotic aorta: implications for atherogenesis and vascular development. Am J Pathol 1998;152: 913-923.

49 Schwartz SM, Murry CE: Proliferation and the monoclonal origins of atherosclerotic lesions. Ann Rev Med 1998;49:437-460.

50 Bogliolo M, Izzotti A, De Flora S, Carli C, Abbondandolo A, Degan P: Detection of the '4,977 bp' mitochondrial DNA deletion in human atherosclerotic lesions. Mutagenesis 1999; 14:77-82.

51 Mueller EE, Eder W, Ebner S, Schwaiger E, Santic D, Kreindl T, Stanger O, Paulweber B, Iglseder B, Oberkofler H, Maier R, Mayr JA, Krempler F, Weitgasser R, Patsch W, Sperl W, Kofler B: The mitochondrial T16189C polymorphism is associated with coronary artery disease in Middle European populations. PLoS One 2011;6:e16455.

52 Sazonova M, Budnikov E, Khasanova Z, Sobenin I, Postnov A, Orekhov A: Studies of the human aortic intima by a direct quantitative assay of mutant alleles in the mitochondrial genome. Atherosclerosis 2009;204:184-190.

53 Sobenin IA, Sazonova MA, Postnov AY, Bobryshev YV, Orekhov AN: Mitochondrial mutations are associated with atherosclerotic lesions in the human aorta. Clin Dev Immunol 2012;2012:832464.

-54 Sobenin IA, Sazonova MA, Postnov AY, Bobryshev YV, Orekhov AN: Changes of mitochondria in atherosclerosis: possible determinant in the pathogenesis of the disease. Atherosclerosis 2013;227:283-288.

55 Sobenin IA, Sazonova MA, Ivanova MM, Zhelankin AV, Myasoedova VA, Postnov AY, Nurbaev SD, Bobryshev YV, Orekhov AN: Mutation C3256T of mitochondrial genome in white blood cells: novel genetic marker of atherosclerosis and coronary heart disease. PLoS One 2012;7:e46573.

56 Sobenin IA, Sazonova MA, Postnov AY, Salonen JT, Bobryshev YV, Orekhov AN: Association of mitochondrial genetic variation with carotid atherosclerosis. PLoS One 2013; 8:e68070 